\title{
Active diffusion limited reactions
}

Cite as: J. Chem. Phys. 150, 154902 (2019); https://doi.org/10.1063/1.5081125

Submitted: 14 November 2018 . Accepted: 25 March 2019 . Published Online: 15 April 2019

Tanwi Debnath, Pulak K. Chosh, Yunyun Li (D), Fabio Marchesoni (D), and Franco Nori (D)

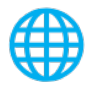




\title{
Active diffusion limited reactions
}

\author{
Cite as: J. Chem. Phys. 150, 154902 (2019); doi: 10.1063/1.5081125 \\ Submitted: 14 November 2018 - Accepted: 25 March 2019 • \\ Published Online: 15 April 2019
}

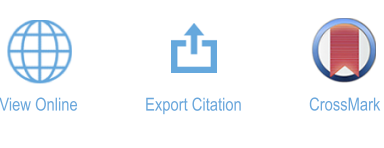

Tanwi Debnath, ${ }^{2}$ Pulak K. Ghosh, ${ }^{2}$ Yunyun Li, ${ }^{3, a)}$ (D) Fabio Marchesoni, ${ }^{3,4}$ (D) and Franco Nori ${ }^{5,6}$

\begin{abstract}
AFFILIATIONS
${ }^{1}$ Department of Chemistry, University of Calcutta, Kolkata 700009, India

${ }^{2}$ Department of Chemistry, Presidency University, Kolkata 700073, India

${ }^{3}$ Center for Phononics and Thermal Energy Science, School of Physics Science and Engineering, Tongji University, Shanghai 200092, People's Republic of China

4Dipartimento di Fisica, Università di Camerino, I-62032 Camerino, Italy

${ }^{5}$ Theoretical Quantum Physics Laboratory, RIKEN Cluster for Pioneering Research, Wako-shi, Saitama 351-0198, Japan

${ }^{6}$ Department of Physics, University of Michigan, Ann Arbor, Michigan 48109-1040, USA
\end{abstract}

Note: This article is part of the Special Topic "Chemical Physics of Active Matter" in J. Chem. Phys.

a) Author to whom correspondence should be addressed: yunyunli@tongji.edu.cn

\begin{abstract}
We investigate the one- and two-dimensional diffusion limited reactions $\mathrm{A}+\mathrm{A} \rightarrow 0$ and $\mathrm{A}+\mathrm{B} \rightarrow 0$ with $\mathrm{A}$ active Janus particles and $\mathrm{B}$ passive particles in thermal equilibrium. We show that by increasing the self-propulsion time of the A particles, the reactant densities decay faster, at least for time transients of potential interest for chemical applications, e.g., to develop smart drug delivery protocols. Asymptotic and transient density decays obey power laws with exponents that depend on the actual annihilation reaction and its dimensionality.
\end{abstract}

Published under license by AIP Publishing. https://doi.org/10.1063/1.5081125

\section{INTRODUCTION}

Artificial microswimmers are active particles capable of autonomous propulsion. ${ }^{1-3}$ Their propulsion mechanism, which is often presented as a biomimetic counterpart of cellular motility, ${ }^{4}$ is a stationary nonequilibrium process triggered by the particles themselves. Owing to a built-in functional asymmetry, ${ }^{5-8}$ they harvest kinetic energy from their environment, for instance, by inducing local (electric, ${ }^{6}$ thermal, ${ }^{9}$ or chemical ${ }^{10}$ ) gradients in the suspension medium (self-phoresis). The simplest class of artificial swimmers investigated in the literature is the so-called Janus particles (JP), mostly spherical colloidal particles with two differently coated hemispheres or "faces." A different face functionalization determines a peculiar dipolar symmetry, which makes the axial propulsion of these particles possible.

Among the prominent applications of artificial microswimmers is their pharmaceutical usage $e^{6}$ to control chemical reactions, either as motile catalysts or reactant carriers, in the context of recently proposed smart drug delivery systems. ${ }^{14}$ Since self-propelled particles undergo persistent Brownian motion with finite correlation time (also termed active Brownian motion), one expects that the chemical processes they initiate are governed in time and space by their "diffusive" properties. Indeed, a single molecular reaction takes place only after the involved active agent has diffused close to a reactant molecule, while the reaction itself is assumed to occur instantaneously. We refer to this class of processes as active diffusion limited reactions (DLR) or active DLR for brevity.

Diffusion limited reactions in low dimensions and in numerous constrained geometries exhibit anomalous behavior, in the sense that their kinetics is not governed by the classical macroscopic laws of mass action but rather by the nonequilibrium dynamics of the reactants. ${ }^{15-21}$ For instance, in the bimolecular DLR, $A+B \rightarrow 0$, if the initial densities of the $\mathrm{A}$ and $\mathrm{B}$ reactants are the same, in an infinite volume of Euclidean dimensions $d \leq 4$, these densities decay "asymptotically" with time $t^{-\alpha}$, with $\alpha=d / 4 .^{18,19}$ This result is in apparent contrast with the exponent $\alpha=1$ predicted by the classical rate law. Another paradigmatic DLR that exhibits anomalous kinetics is the bimolecular reaction $\mathrm{A}+\mathrm{A} \rightarrow 0$, where the reactant density decays with time also according to a power law but with exponent $\alpha=d / 2$.

An exponent smaller than that predicted by the classical mass law, $\alpha<1$, is generally attributed to the formation of persistent spatio-temporal structures in the reactant distributions. ${ }^{22,23}$ Following this interpretation, one may expect that introducing some 
mixing mechanism may suppress such "transient" structures and, therefore, increase the decay exponent $\alpha$.

As argued in Ref. 22, inertia in the reactant diffusive dynamics provides an analytically and numerically manageable example of such a mixing mechanism. Indeed, on decreasing the viscous constant of the A and B particles, the exponent $\alpha$ increases from its known diffusive value up to substantially larger values peculiar to the ballistic regime. Here, by inertia we mean that each diffusing particle retains memory of its past trajectory for a time of the order of the reciprocal of its viscous constant. Recalling that an active particle undergoes persistent Brownian motion with correlation time that depends on its self-propulsion mechanism, ${ }^{24}$ one is led to think that DLRs involving active particles are characterized by $\alpha$ exponents also depending on their self-propulsion parameters. That would make $\alpha$ easier to tune, in view of practical applications, whereas the same result could be hardly achieved by manipulating the particle viscous constant.

The content of this paper is organized as follows. In Sec. II, we introduce the stochastic equations for diffusing active and passive particles in $\mathrm{d}=1$ (1D) and $\mathrm{d}=2$ dimensions (2D).

Active particles are modeled as JPs with constant axial speed and randomly fluctuating orientation. We then limit our study to the two archetypal bimolecular DLRs mentioned above, $\mathrm{A}+\mathrm{A} \rightarrow 0$ and $\mathrm{A}+\mathrm{B} \rightarrow 0$, avoiding all unnecessary complications widely discussed in the DLR literature. In Sec. III, we summarize the results of Ref. 22 for both A and B passive particles, with particular attention to the effects of inertia on the time decay of the particle densities. In Sec. IV, we investigate the same reactions except that now the A particles are active and the B particles are passive. In both Secs. III and IV, we consider $1 \mathrm{D}$ and $2 \mathrm{D}$ particle mixtures, separately. In Sec. V, we analyze in some details the spatial distributions of the $\mathrm{A}$ and $\mathrm{B}$ particles for the $\mathrm{A}+\mathrm{B} \rightarrow 0$ reactions of Sec. IV and show how self-propelling A particles do exert a mixing action on the A-B mixture. Finally, in Sec. VI, we comment on the potential applications of active DRL's.

\section{MODEL}

In the present work, we numerically simulated mixtures of $n_{A}$ A-particles and $n_{B}$ B-particles with $n_{A}=n_{B}$. The mixtures were confined to a segment of length $L$ for $d=1$ and a square of area $L^{2}$ for $d=2$. Accordingly, the reactant densities were $\rho_{A, B}=n_{A, B} / L^{d}$, with total mixture density $\rho_{A+B}=\rho_{A}+\rho_{B}$. The active particles considered here are two-dimensional JPs, that is, two-faced disks of (small) radius $r_{0}$, corresponding to (low) packing fractions, $\phi=2 r_{0} \rho_{A+B}$ for $d=1$ and $\phi=\pi r_{0}^{2} \rho_{A+B}$ for $d=2$. We have already modeled the JP dynamics in Refs. 25-27; here, our simulation code was modified to account for possible inertial effects in the spirit of Ref. 22.

The free dynamics of a single diffusing particle of unit mass $(m=1)$ obeys the Langevin equations ${ }^{28,29}$

$$
\begin{aligned}
& \ddot{x}=-\gamma \dot{x}+\gamma v_{0} \cos \theta+\xi_{x}(t), \\
& \ddot{y}=-\gamma \dot{y}+\gamma v_{0} \sin \theta+\xi_{y}(t), \\
& \dot{\theta}=\xi_{\theta}(t),
\end{aligned}
$$

where the particle center of mass, $\mathbf{r}$, is confined to the plane $(x, y)$, subject to an isotropic viscous damping with constant $\gamma$ and equilibrium thermal fluctuations represented by the Gaussian noises $\xi_{i}(t)$, with $\left\langle\xi_{i}(t)\right\rangle=0$ and $\left\langle\xi_{i}(t) \xi_{j}(0)\right\rangle=2 \gamma k T \delta_{i j} \delta(t)$ for $i=x, y$. As shown in Fig. 1(a), active JPs propel themselves with constant speed $v_{0}$ and orientation $\theta$ with respect to the $x$ axis. Of course, when simulating a passive particle, one sets $v_{0}=0$. $1 \mathrm{D}$ mixtures were confined to the $x$ axis so that the Langevin equation for $y$ was ignored. The fluctuations of the propulsion velocity are modeled by the Gaussian noise $\xi_{\theta}(t)$, with $\left\langle\xi_{\theta}(t)\right\rangle=0$ and $\left\langle\xi_{\theta}(t) \xi_{\theta}(0)\right\rangle$ $=2 D_{r} \delta(t)$, where $\tau_{r}=1 / D_{r}$ is the correlation time of the ensuing persistent Brownian motion $\left(D_{r}\right.$ quantifying the angular diffusion of $\mathbf{v}_{0}$ ). In the case of active particles, for asymptotically large times, $t \gg \tau_{r}, 1 / \gamma$, self-propulsion contributes an additional amount of $D_{s}=v_{0}^{2} / 2 D_{r}$ to the thermal diffusivity, $D_{0}=k T / \gamma$. Passive particles are subject to thermal diffusivity only. We treated all noise sources in Eq. (1) as independently tunable, although, strictly speaking, thermal and orientational fluctuations may be statistically correlated (see, e.g., Ref. 30). More importantly, the propulsion and fluctuation parameters used in our simulations are experimentally accessible, as apparent on expressing times in seconds and lengths in microns. ${ }^{3}$

When simulating a confined particle mixture, one has to specify the collisional processes as well: (i) Non-annihilating particle collisions were assumed to be elastic. Note that, in $1 \mathrm{D}$, particles of the same species are non-passing and thus tend to form local single files of reduced diffusivity; ${ }^{16,32}$ (ii) collisions against the walls of the container are always elastic; under this condition, walls can be replaced by periodic boundary conditions; ${ }^{25}$ (iii) in a first approximation, the short-range interactions among active and/or passive particles and the hydrodynamics of self-propulsion are neglected.
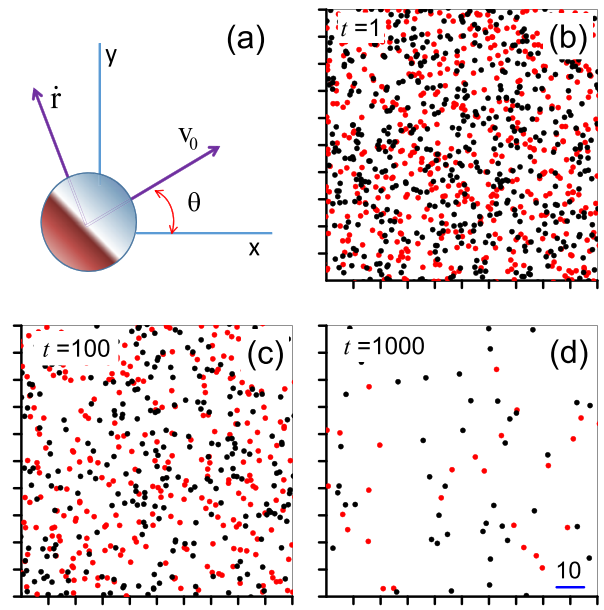

FIG. 1. (a) Two dimensional active JP model (see text). The dynamical and the self-propulsion velocities are represented by distinct vectors. [(b)-(d)] Snapshots of a $2 \mathrm{D}$ A-B mixture at three different times in a $100 \times 100$ sampling region. The simulation box with side $L=1000$ contained $n_{A}=5 \times 10^{4}$ active $A$ particles (JP, red) and $n_{B}=5 \times 10^{4}$ passive $B$ particles (black, circle size not to scale). All particles are disks of radius $r_{0}=0.05$ to ensure a very low initial box packing fraction. Upon collision, the $\mathrm{A}-\mathrm{B}$ pairs annihilate, $A+B \rightarrow 0$, while all other collisions are elastic. Reflecting and periodic conditions at the box boundaries return the same decay law. 
The stochastic differential equation (1) was numerically integrated by means of a standard Mil'shtein scheme. ${ }^{31}$ To avoid biasing the simulation outcome with unwanted spatial structures, we imposed random initial conditions, that is, uniform spatial distributions, Gaussian velocity distributions with temperature $T$, and uniform angular distributions of the vector $\mathbf{v}_{0}$ for active particles. All stochastic averages were taken over not less that 1000 realizations of each mixture annihilation.

We discuss now the characteristic time scales of the active DLRs under investigation. The diffusive dynamics of Eq. (1) introduces four rates: two are related to thermal relaxation (the viscous damping constant, $\gamma$, and the diffusive rate, $k T / \gamma l^{2}$ ) and two more are associated with the self-propulsion mechanism of the active particles (the reciprocal of the persistence time or angular rate, $D_{r}$, and the reciprocal of the free self-propulsion time, $\left.v_{0} / l\right)$. Here, $l$ denotes an appropriate mixture length scale, the most obvious choice being $l=\rho^{-1 / d}$, where $\rho$ denotes a reactant density, and $l \gg r_{0}$. To make contact with the active JPs used in real experiments, ${ }^{30}$ we assumed low Reynolds numbers, that is, large $\gamma, \gamma / D_{r}$ $\gg 1$, and long observation times, $\gamma t \gg 1$. Moreover, due to the strong advective character of self-motility, we restricted our analysis to high Péclet numbers, $P e_{r}=\gamma r_{0} v_{0} / k T \gg 1$. Finally, the bimolecular chemical reactions investigated here are actually governed by active diffusion only under the additional condition $D_{s} \gg D_{0}$, that is, for $\gamma v_{0}^{2} / D_{r} k T \gg 1$.

The diffusion controlled annihilation of the mixture reactants occurs within the time interval $\left(t_{0}, t_{\infty}\right)$, where (i) $t_{0}$ is the average time two neighboring reactant molecules take to collide. For a pair of passive particles, $t_{0}$ is of the order of $l^{2} / D_{0}$, i.e., the reciprocal of the diffusive rate, $k T / \gamma l^{2}$, introduced above. Of course, for pair collisions involving an active particle, it is shorter, being proportional to $l^{2} / D_{s}$; (ii) $t_{\infty}$ marks the end of the process, after all reactant pairs have disappeared. This time $t_{\infty}$ depends on the initial reactant density and restricts our ability to numerically assess the transient nature in the mixture decay dynamics.

\section{THE ROLE OF INERTIA}

We first report our simulation results for the bimolecular DLRs, $\mathrm{A}+\mathrm{A} \rightarrow 0$ and $\mathrm{A}+\mathrm{B} \rightarrow 0$, in the absence of self-propulsion, $v_{0}=0$ (A and $B$ particles are both passive, though chemically distinct $\left.{ }^{15,16}\right)$, but over a large range of $\gamma$ bridging the underdamped and overdamped limits. We briefly revise the results of Ref. 22 proving that inertia actually exerts a mixing action on the mixture by quickly suppressing emerging spatio-temporal structures and thus speeding up the time decay of the reactant densities. In panels (a) and (b) of Fig. 2, we illustrate the $\rho(t)$ time decay, respectively, for $\mathrm{A}+\mathrm{A} \rightarrow 0$ and $\mathrm{A}+\mathrm{B} \rightarrow 0$ in $1 \mathrm{D}$; the $\rho(t)$ curves for the corresponding $2 \mathrm{D}$ reactions are displayed in panels $(\mathrm{c})$ and $(\mathrm{d})$.

For large $\gamma$, we recover the diffusive behavior anticipated in Sec. I: The time decay of $\rho(t)$ follows an asymptotic power law, $\rho(t) \sim t^{-\alpha}$, with $\alpha=d / 2$ for $\mathrm{A}+\mathrm{A} \rightarrow 0$ [(a) and (c)] and $\alpha=d / 4$ for $\mathrm{A}+\mathrm{B} \rightarrow 0[(\mathrm{~b})$ and $(\mathrm{d})]$.

At very small $\gamma$, we correctly reproduce the faster decay law, $\rho(t)$ $\sim t^{-\beta}$, with $\beta \geq \alpha$, first reported in Ref. 22. For the $\mathrm{A}+\mathrm{B} \rightarrow 0$ reaction, a simple generalization ${ }^{22}$ of the fluctuating density argument of Ref. 19 suggests that $\beta=2 \alpha$, namely, $\beta=d / 2$. We confirm the
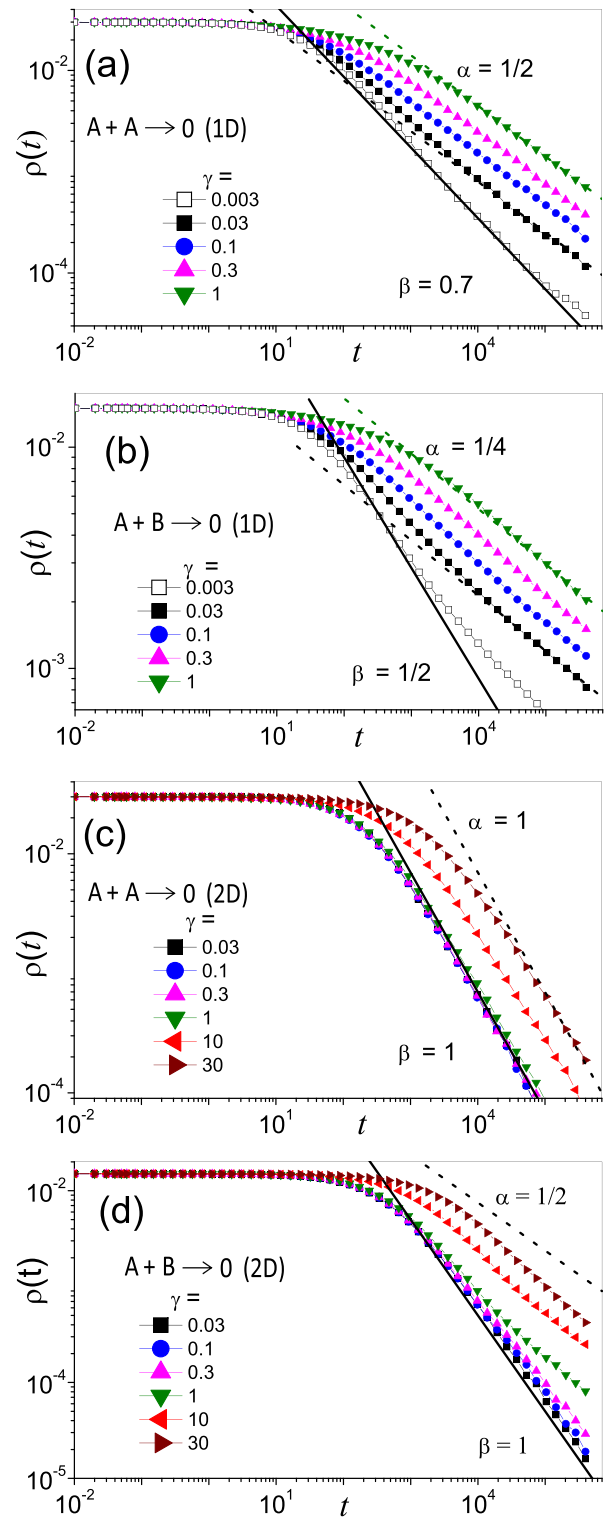

FIG. 2. Time decay of the A particle density $\rho(t)=\rho_{A}(t)$ for $[(a)$ and $(c)] A+A$ $\rightarrow 0$, [(b) and (d)] A + B $\rightarrow 0$, [(a) and (b)] $d=1$, and [(c) and (d)] $d=2$. Here, $\gamma$ varies from 0.003 up to 30 (see legends). A and B particles are both passive with $v_{0}=0$. All curves seem to decay with power laws $\rho(t) \sim t^{-\alpha}$ (high $\gamma$, diffusive limit) and $\rho(t) \sim t^{-\beta}$ (low $\gamma$, ballistic limit). The fitted exponents are also reported in the legends. The curve for $\gamma=30$ in (d) approaches the expected decay slope only for larger $t$ (not shown for graphical reasons). While the $t^{-\alpha}$ decay seems to set in asymptotically, the $t^{-\beta}$ decay is compatible with a transient effect extending beyond the accessible observation time, $t>t_{\infty}$. Simulation parameters: (a) $n_{A}$ $=3 \times 10^{4}, L=10^{6}$; (b) $n_{A}=n_{B}=1.5 \times 10^{4}, L=10^{6}$; (c) $n_{A}=3 \times 10^{4}, L=10^{3} ;$ (d) $n_{A}=n_{B}=1.5 \times 10^{4}, L=10^{3}$. Other simulation parameters: $r_{0}=0.05, k T=0.2$, $m=1$; all lengths are expressed in arbitrary units.

estimate of $\beta$, at least within the numerically accessible time interval $\left(t_{0}, t_{\infty}\right)$. Of course, we cannot rule out the possibility that such a faster decay is a transient effect only, with $\beta$ eventually approaching $\alpha$ at even larger times, $t \gg t_{\infty}$. Verifying this hypothesis 
would require simulating much larger mixtures, which lies beyond our present computational capabilities.

In $2 \mathrm{D}$, the $\mathrm{A}+\mathrm{A} \rightarrow 0$ reaction is known ${ }^{16}$ to obey the classical mass law, with $d=2$ as its critical dimension, in both dynamical limits. The numerical results of Fig. 2(c) validate this prediction, that is, $\beta=1$.

Regarding the ballistic regime of the $\mathrm{A}+\mathrm{A} \rightarrow 0$ reaction in $1 \mathrm{D}$, there is no consensus on the value of $\beta$ (see Ref. 22 for a detailed discussion). The proposed estimates range between $2 / 3$ and 0.805 , whereas numerical simulations for a noiseless mixture yields $\beta=0.79$. For the lowest $\gamma$ values considered here, Fig. 2(a), a best fit returned $\beta=0.7$.

The most important conclusion of this section is that "the Langevin dynamics of Eq. (1) bridges the ballistic and diffusive decay regimes with $\gamma$ acting as an effective control parameter."

\section{THE ROLE OF ACTIVE DIFFUSION}

In Fig. 3, we illustrate simulation results for the active DLRs $\mathrm{A}+\mathrm{A} \rightarrow 0$, panels (a) and (c), and $\mathrm{A}+\mathrm{B} \rightarrow 0$, panels (b) and (d). The main conclusions of our numerical investigation can be summarized as follows:

(i) Similar to inertia in Sec. III, the persistent Brownian motion of the active A particles suppresses the retarding effects of spatio-temporal fluctuations in the mixture configuration. Indeed, a general feature of all panels of Fig. 3 is the fast time decay of $\rho(t)$ for small $D_{r}$ (large $\tau_{r}$ ). This applies also for large $\gamma$, where we know from Fig. 2 that inertia effects are negligible.

(ii) The faster decays of $\rho(t)$ at low $D_{r}$, quantified by the $\beta$ exponent, are transient effects. Indeed, almost all curves plotted in Fig. 3 hint at approaching the corresponding diffusive decay law, $t^{-\alpha}$, already for $t<t_{\infty}$. [Exceptions are mentioned in item (iv).] This is not surprising because, as anticipated in Sec. II, for $t \gg \tau_{r}$ active Brownian motion is indistinguishable from a regular Brownian motion with enhanced diffusivity, $D_{0}+D_{s}$. At sufficiently low reactant density, that is, for large spatial separation of the reactant pairs, the self-propulsion memory of the pair constituents becomes negligible.

(iii) Persistence effects in the self-propulsion mechanism imply that, at high Péclet numbers, the motion of the A particles is ballistic for time interval of the order of $\tau_{r}$. This reflects in the values of the $\beta$ exponents of panels (c) and (d), which coincide with those of the corresponding panels (c) and (d) of Fig. 2. Stated otherwise, in 2D for long self-propulsion times of the A particles, the decay of both DLRs starts out ballistically.

(iv) Deviations from the general picture of items (ii) and (iii) appear at low $\gamma$ and $D_{r}$ due to the interplay of inertia and persistent activation effects. In that dynamical regime, $t_{\infty}$ was not large enough to assess the asymptotic decay of the mixture density. We notice that, for practical applications, such a regime is of lesser interest as active JPs operate at low Reynolds numbers.

On inspecting Figs. 3(a) and 3(b), it becomes apparent that in $1 \mathrm{D}$ the $\beta$ exponents are larger than their counterparts in Fig. 2, namely, larger than predicted for the ballistic regime of Sec. III. This
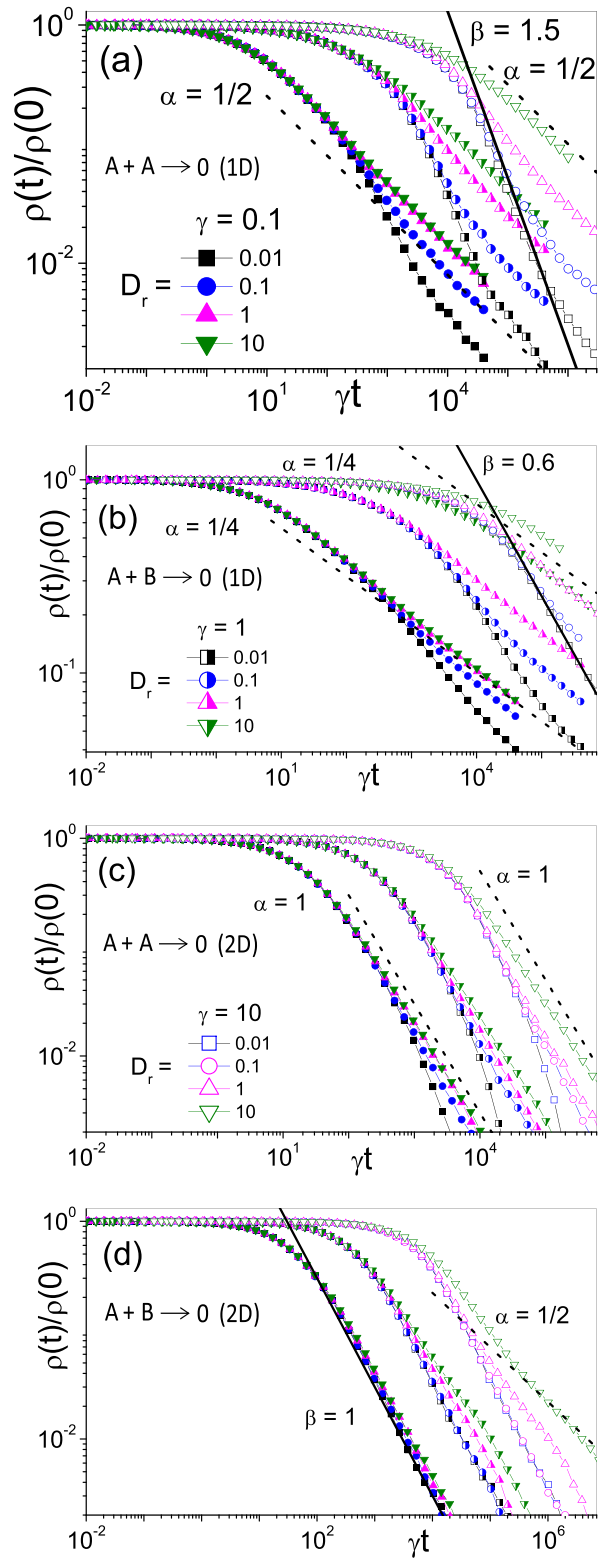

FIG. 3. Time decay of the A particle density $\rho(t)=\rho_{A}(t)$ for $[(a)$ and $(c)] A+A \rightarrow 0$ [(b) and (d)] A + B $\rightarrow 0$, [(a) and (b)] $d=1$, and [(c) and (d) $] d=2$ and different $\gamma$. Here, $\mathrm{A}$ are active particles with $v_{0}=1$ and different angular rates, $D_{r}$ (see legend); $B$ are passive particles. The exponents $\alpha$ and $\beta$ of the diffusive (large $D_{r}$ ) and transient ballistic (low $D_{r}$ ) time decays are written next to the dashed and solid lines, respectively; $\alpha$ and $\beta$ do not change by halving or doubling the mixture packing fraction. Simulation parameters: (a) $n_{A}=3 \times 10^{4}, L=10^{6}$; (b) $n_{A}=n_{B}$ $=5 \times 10^{4}, L=10^{6}$; (c) $n_{A}=3 \times 10^{4}, L=10^{3} ;$ (d) $n_{A}=n_{B}=5 \times 10^{4}, L=10^{3}$. Other simulation parameters: $r_{0}=0.05, k T=0.2, m=1$; all length are expressed in arbitrary units. Legends in (a)-(c) apply to all four panels.

effect is due to having constrained a persistent Brownian motion along a line.

For the $\mathrm{A}+\mathrm{A} \rightarrow 0$ reaction, we propose the following simple interpretation. Being confined to the $x$ axis, the active A particles 
move either right $(\mathrm{R})$ or left $(\mathrm{L})$ with velocities $\pm \bar{v}_{0}$. Their average $1 \mathrm{D}$ speed is smaller that $v_{0}$. Assuming uniform unconstrained angular fluctuations (no wall effects), like in Eq. (1), $\bar{v}_{0}=(2 / \pi) v_{0}$. The distribution of the $\mathrm{R}(\mathrm{L})$ oriented particles is uniformly randomized as an effect of direction switches occurring with rate $D_{r}$. Only R-L pairs annihilate (instantaneously) upon collision. In this approximation, the $1 \mathrm{D}$ active $\mathrm{A}+\mathrm{A} \rightarrow 0$ reaction reduces to the dichotomic $\mathrm{A}+\mathrm{B} \rightarrow 0$ reaction of Refs. 23, 33, and 34, with A and B representing, respectively, the $\mathrm{R}$ and $\mathrm{L}$ oriented active JPs. Due to the symmetric dynamics of the system and its initial conditions, $\rho_{L}(0)$ $=\rho_{R}(0)$, the reactant density is predicted to decay asymptotically like

$$
\rho_{\alpha}(t) \sim\left[\rho(0) /\left(\pi v_{0} t\right)\right]^{1 / 2} .
$$

The exponent of this power law, $\alpha=1 / 2$, coincides with the diffusive decay exponent predicted for the $\mathrm{A}+\mathrm{A} \rightarrow 0$ reaction in a $1 \mathrm{D}$ mixture of passive A particles.

However, as noticed in Ref. 23, the mechanisms responsible for these two asymptotic decay laws are different. Indeed, at shorter times, $t \sim \tau_{r}$, the decay process is sensitive to the velocity switching mechanism so that the onset of density fluctuations along the line starts playing a role. Close to equilibrium, it is reasonable to assume ${ }^{18,19}$ particle number fluctuations of the order of $\left(\rho(0) v_{0} \tau_{r}\right)^{1 / 2}$. (We remind that we agreed to treat the $\mathrm{R}$ and $\mathrm{L}$ particles of the same A mixture as different species.) In the presence of such an R and L density unbalance, one predicts ${ }^{33,34}$ a faster $\rho(t)$ decay law, $\rho_{\beta}(t)=\kappa(t) \rho_{\alpha}(t)$, with

$$
\kappa(t)=\left(\tau_{r} / 2 t\right) \exp \left(-t / \tau_{r}\right) .
$$

In conclusion, we expect a fast transient decay at short times with $\beta=3 / 2$, which is consistent with the data of Fig. 3(a). Note that the above argument applies best for large $\gamma$ (no inertia effects) and $D_{r} \ll \gamma$ (strong orientation memory).

To explain the transient density decay of the A-B mixture in Fig. 3(b), we had recourse to a qualitative scaling argument. Note that an A-B mixture consists of two interacting fractions: the stationary self-propelling A particles and the thermally diffusive B particles; a problem not much investigated, not even in 1D. Let us consider the mixture transient dynamics and ask ourselves the following question: How many A-B pairs do survive along the distance, $l_{s}(t)=v_{0} t$, covered by a self-propelling A particle during the initial time interval $t$, with $t \leq \tau_{r}$ ? A qualitative answer is

$$
\rho(t) \sim \rho(0)\left[x_{A A}(t) / l_{s}(t)\right],
$$

where $x_{A A}(t)$ is the ever expanding pair distance ${ }^{16}$

$$
x_{A A}(t) \sim t^{3 / 8} .
$$

As a result, $\rho(t)$ decays with time according to a power law with exponent $\beta=5 / 8$, in fairly good agreement with the data of Fig. 3(b).

\section{REACTANT SPATIAL DISTRIBUTIONS}

The remarkable difference between the time decay of the $1 \mathrm{D}$ and $2 \mathrm{D}$ mixtures is mostly of topological nature. In $1 \mathrm{D}$, diffusing particles collide only with their nearest-neighbors (nonpassing dynamics). This implies that fluctuations of the reactant concentration tend to be long-lived. Such a dimensional constraint was implicitly accounted for in our interpretation of the data of Figs. 3(a) and 3(b). Active DLRs in 2D are more interesting because, first, they are easier to investigate experimentally and, second, the spatial mixing action of the active reactants is not limited by the dimensional constraint mentioned above.

In order to elucidate the mixing action of active particles in an annihilating mixture, we characterized the reactants' spatial distribution by measuring the pair distances $r_{A A}$ for $\mathrm{A}+\mathrm{A} \rightarrow 0$ and $r_{A A}$ and $r_{A B}$ for $\mathrm{A}+\mathrm{B} \rightarrow 0$. We defined the pair distances $r_{A A}$ and $r_{A B}$ as the distance between any tagged $\mathrm{A}$ particle and the closest $\mathrm{A}$ or $\mathrm{B}$ particle, respectively. Distributions of $r \equiv r_{A A}$ for the 2D mixtures of Fig. 3 at different times, $P(r, t)$, are displayed in Fig. 4 .

In sharp contrast with the case of all-passive mixtures, ${ }^{22,23}$ no distinguishable pattern formation emerged during the decay transients (see also the graphic rendering of Fig. 1). We remind that both A-A and A-B mixtures where prepared by initially distributing all their constituents at random inside the simulation box (Poissonian spatial distribution $^{35}$ ). A direct computation showed that at $t=0\left\langle r_{A A}\right\rangle=\left(1 / 2 \rho_{A}\right)^{1 / 2}$ for the A-A mixture, while for the A-B mixture $\left\langle r_{A A}\right\rangle=\left(1 / 2 \rho_{A}\right)^{1 / 2}$ and $\left\langle r_{A B}\right\rangle=\left\langle r_{A A}\right\rangle / 2$. We remind that in our figures $\rho(t)$ is a shorthanded notation for $\rho_{A}$ at time $t$, namely, either the total instantaneous particle density of the A-A mixture or just half the total density of the A-B mixture.

As suggested by the initial pair distance averages, in Figs. 4(c) and $4(\mathrm{~d})$, we rescaled $r$ as

$$
r \rightarrow r / l(t), \text { with } l(t)=[1 / \rho(t)]^{1 / 2} .
$$

For low angular rates, $D_{r}, P(r, t)$ peaks at $r / l(t) \simeq 0.4$ and, more remarkably, there is no appreciable change in the distribution profiles up to large times. In particular, we verified that the initial relations between the averages $\left\langle r_{A A}\right\rangle$ and $\left\langle r_{A B}\right\rangle$ and the density $\rho$ keep holding as the decay process progresses independently of the damping regime. We remind that for $2 \mathrm{D}$ mixtures of passive particles one would expect latticelike and clusterlike structures, respectively, in the diffusive (large $\gamma)^{22}$ and ballistic limit (low $\gamma)$.

For the sake of comparison, we used the approach of Ref. 35 to calculate analytically $P(r, t)$ for $d=2$ and $r_{0} \ll l(0)$. Our result,

$$
P(r, t)=\frac{2 r}{l^{2}(t)} \exp \left(-\frac{r^{2}}{l^{2}(t)}\right),
$$

with $\bar{l}(t)=[\pi \rho(t)]^{-1 / 2}$, reproduces quite closely the numerical distributions of Figs. 4(c) and 4(d) - small deviations are attributable to the finite particle size. Not surprisingly, in $1 \mathrm{D}$, our curves for $P(r, t)$ are well approximated by Erlang distributions (not shown). ${ }^{16}$

We interpret these results as strong evidence that the A particles remain quite uniformly distributed in the simulation box during the entire transient of the annihilation process. A similar behavior was detected also for the time dependent distributions of $r_{A B}$ (not shown), which led us to conclude that active particles act like effective mixture stirrers.

Finally, another interesting feature of the mixtures' spatial configurations emerges by comparing Figs. 4(a) and 4(b), where the distances $r$ have now been rescaled by the fixed preparation length, $l(0)$. At large $t$, the $P(r, t)$ maxima appear to move to higher $r$ values as the reactants annihilate, with speed proportional to $\rho(0)$. Note 

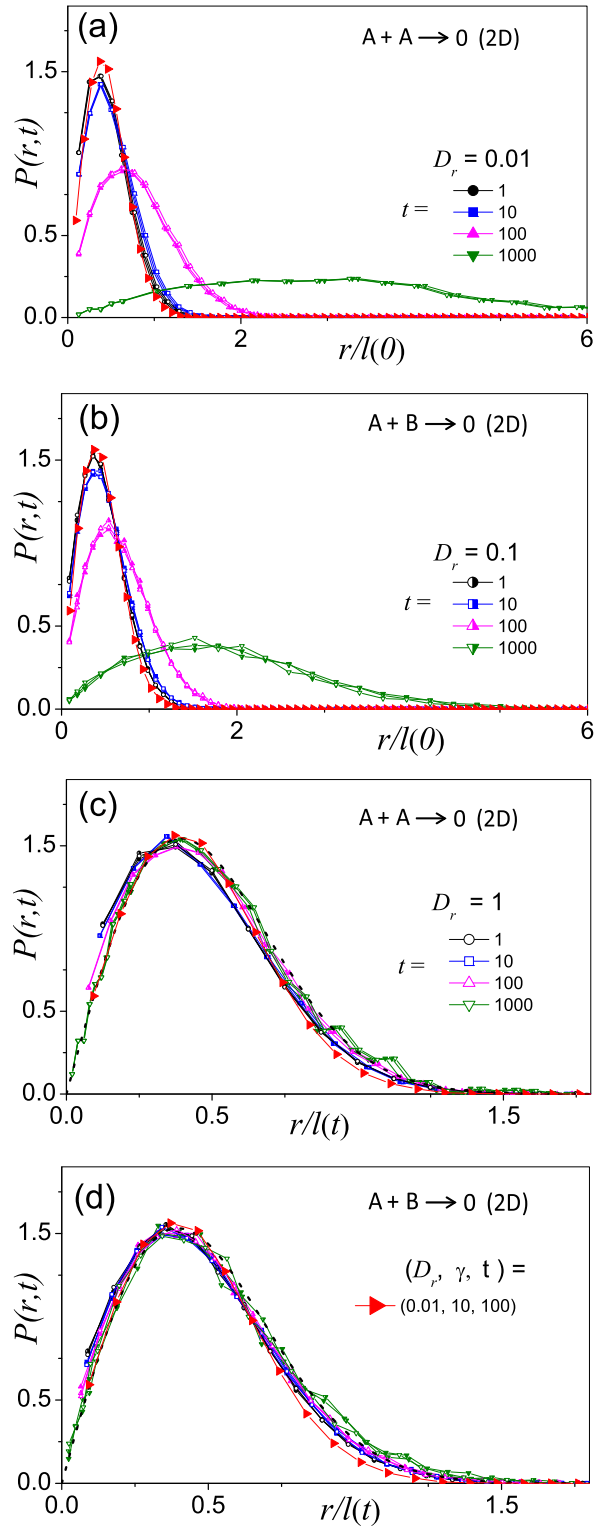

FIG. 4. Distribution of $r \equiv r_{A A}$ at different times for $A+A \rightarrow 0[(a)$ and $(c)]$ and $A+B$ $\rightarrow 0[(\mathrm{~b})$ and $(\mathrm{d})]$ in 2D. Here, $A$ are active particles with $v_{0}=1$ and $D_{r}=0.01$ and $B$ are passive particles. The rescaling length $/$ is a function of time, $I(t)=[1 / \rho(t)]^{1 / 2}$, with $\rho(t)=\rho_{A}(t)$. Upon using the time dependent rescaling length $I(t)$, all three data sets of (a) and (b) collapse on a unique curve, respectively, in (c) and (d). The dashed curves in (c) and (d) represent the relevant theoretical predictions, Eq. (2). Simulation parameters: $[(\mathrm{a})$ and $(\mathrm{c})] n_{A}=10^{5}$ and $[(\mathrm{b})$ and (d) $] n_{A}=n_{B}=5 \times 10^{4}$. Moreover, $L=10^{3}, r_{0}=0.05, k T=0.2, m=1, \gamma=0.1[(\mathrm{a})$-(c)] and 10 (d), with all lengths given in arbitrary units. Legends in (a)-(d) apply to all four panels.

that in Fig. 4(a) the initial value of $\rho_{A}$ is twice than in Fig. 4(b). The two active DLRs are actually controlled by a diffusive mechanism, as to be expected for $D_{r} t \gg 1$. In fact, the r.m.s. of the reactant pair separation appears to grow with time proportional to the product of the reactant densities.

\section{CONCLUSIONS}

In this paper, we investigated the role of active diffusive particles in the broad context of the so-called diffusion limited chemical reactions. We restricted our presentation to the archetypal reactions of pair annihilation, $\mathrm{A}+\mathrm{A} \rightarrow 0$ and $\mathrm{A}+\mathrm{B} \rightarrow 0$, in one and two dimensions. Moreover, we considered the simplest realization of an artificial microswimmer consisting of an active Janus particle. Most of our results are based on simulation data and phenomenological arguments.

Despite all the simplifications listed above, we gathered evidence of the capability of artificial microswimmers to accelerate the chemical kinetics they assist, either as catalysts or reactant carriers. Such a capability grows prominently in low dimensional or strongly constrained geometries. In particular, we proved that self-propellers have a mixing or stirring effect on the configurational fluctuations occurring in the reactant mixture, which speeds up the annihilation processes.

From a more fundamental point of view, we highlighted the role of memory effects in the diffusive mechanism controlling this class of chemical reactions. Such effects, scarcely addressed in the specialized literature, ${ }^{36}$ become now experimentally accessible by making use of active microparticles or nanoparticles since these diffuse with correlation times governed by the same external mechanisms also regulating their motility.

\section{ACKNOWLEDGMENTS}

We thank RIKEN's RICC for computational resources. Y. Li is supported by the NSF China under Grant No. 11875201. P.K.G. is supported by SERB Start-up Research Grant (Young Scientist) No. YSS/2014/000853 and UGC-BSR Start-Up Grant No. F.30-92/2015. T.D. thanks UGC, New Delhi, India, for support through a Junior Research Fellowship. F.N. is supported in part by the MURI Center for Dynamic Magneto-Optics via the Air Force Office of Scientific Research (AFOSR) (Grant No. FA9550-14-1-0040), the Army Research Office (ARO) (Grant No. W911NF-18-1-0358), the Asian Office of Aerospace Research and Development (AOARD) (Grant No. FA2386-18-1-4045), the Japan Science and Technology Agency (JST) (Q-LEAP program, ImPACT program, and CREST Grant No. JPMJCR1676), the Japan Society for the Promotion of Science (JSPS) (JSPS-RFBR Grant No. 17-52-50023 and JSPS-FWO Grant No. VS.059.18N), the RIKEN-AIST Challenge Research Fund, and the John Templeton Foundation.

\section{REFERENCES}

${ }^{1}$ F. Schweitzer, Brownian Agents and Active Particles (Springer, Berlin, 2003).

${ }^{2}$ Janus Particle Synthesis, Self-Assembly and Applications, edited by S. Jiang and S. Granick (RSC Publishing, Cambridge, 2012).

${ }^{3}$ A. Walther and A. H. E. Müller, "Janus particles: Synthesis, self-assembly, physical properties, and applications,” Chem. Rev. 113, 5194 (2013).

${ }^{4}$ M. J. McBride, "Bacteria gliding motility: Multiple mechanisms for cell movement over surfaces," Annu. Rev. Microbiol. 55, 49 (2001); H. C. Berg, E. Coli in Motion (Springer, New York, 2013).

${ }^{5}$ J. Elgeti, R. G. Winkler, and G. Gompper, "Physics of microswimmers, single particle motion and collective behavior: A review," Rep. Prog. Phys. 78, 056601 (2015).

${ }^{6}$ S. Sengupta, M. E. Ibele, and A. Sen, "Fantastic voyage: Designing self-powered nanorobots,” Angew. Chem., Int. Ed. 51, 8434 (2012). 
${ }^{7} \mathrm{X}$. Wang et al., "Visible light actuated efficient exclusion between plasmonic $\mathrm{Ag} / \mathrm{AgCl}$ micromotors and passive beads," Small 14, 1802537 (2018).

${ }^{8} \mathrm{X}$. Wang et al., "High-motility visible light-driven $\mathrm{Ag} / \mathrm{AgCl}$ Janus micromotors," Small 14, 1803613 (2018).

${ }^{9}$ H. R. Jiang, N. Yoshinaga, and M. Sano, "Active motion of a Janus particle by selfthermophoresis in a defocused laser beam," Phys. Rev. Lett. 105, 268302 (2010).

${ }^{10}$ Y. Hong, N. M. K. Blackman, N. D. Kopp, A. Sen, and D. Velegol, “Chemotaxis of nonbiological colloidal rods," Phys. Rev. Lett. 99, 178103 (2007).

${ }^{11}$ J. P. Hernandez-Ortiz, C. G. Stoltz, and M. D. Graham, "Transport and collective dynamics in suspensions of confined swimming particles," Phys. Rev. Lett. 95, 204501 (2005)

${ }^{12}$ R. Golestanian, T. B. Liverpool, and A. Ajdari, "Designing phoretic micro- and nano-swimmers," New J. Phys. 9, 126 (2007).

${ }^{13}$ R. Golestanian, T. B. Liverpool, and A. Ajdari, "Propulsion of a molecular machine by asymmetric distribution of reaction products," Phys. Rev. Lett. 94, 220801 (2005).

${ }^{14}$ Smart Drug Delivery System, edited by A. D. Sezer (IntechOpen, 2016).

${ }^{15} \mathrm{E}$. Kotomin and V. Kuzovkov, Modern Aspects of Diffusion-Controlled Reactions (Elsevier, Amsterdam, 1996).

${ }^{16}$ D. ben-Avraham and S. Havlin, Diffusion and Reactions in Fractals and Disordered Systems (Cambridge University Press, Cambridge, 2000).

${ }^{17}$ A. Blumen, J. Klafter, and G. Zumofen, in Optical Spectroscopy of Glasses, edited by I. Zschokke (Reidel, Dordrecht, 1986).

${ }^{18}$ A. A. Ovchinnikov and Y. B. Zeldovich, "Role of density fluctuations in bimolecular reaction kinetics," Chem. Phys. 28, 215 (1978).

${ }^{19} \mathrm{D}$. Toussaint and F. Wilczek, "Particle-antiparticle annihilation in diffusive motion," J. Chem. Phys. 78, 2642 (1983).

${ }^{20}$ D. C. Torney and H. M. McConnell, "Diffusion-limited reactions in one dimension," J. Chem. Phys. 87, 1941 (1983).

${ }^{21} \mathrm{~K}$. Kang and S. Redner, "Fluctuation-dominated kinetics in diffusion-controlled reactions," Phys. Rev. A 32, 435 (1985).

${ }^{22}$ A. H. Romero, A. M. Lacasta, J. M. Sancho, and K. Lindenberg, "Numerical study of $\mathrm{A}+\mathrm{A} \rightarrow 0$ and $\mathrm{A}+\mathrm{B} \rightarrow 0$ reactions with inertia," J. Chem. Phys. 127, 174506 (2007).
${ }^{23}$ W. S. Sheu and S. C. Wang, "Effects of velocity relaxation on the anomalous kinetics of a one-dimensional A + A $\rightarrow 0$ reaction," Phys. Rev. E 78, 046101 (2008).

${ }^{24}$ P. K. Ghosh, Y. Li, G. Marchegiani, and F. Marchesoni, "Communication: Memory effects and active Brownian diffusion," J. Chem. Phys. 143, 211101 (2015).

${ }^{25}$ P. K. Ghosh, V. R. Misko, F. Marchesoni, and F. Nori, "Self-propelled Janus particles in a ratchet: Numerical simulations," Phys. Rev. Lett. 110, 268301 (2013).

${ }^{26}$ Y. Li, P. K. Ghosh, F. Marchesoni, and B. Li, "Manipulating chiral microswimmers in a channel," Phys. Rev. E 90, 062301 (2014).

${ }^{27}$ D. Debnath, P. K. Ghosh, Y. Li, F. Marchesoni, and B. Li, "Diffusion of eccentric microswimmers," Soft Matter 12, 2017 (2016).

${ }^{28}$ I. Berdakin, Y. Jeyaram, V. V. Moshchalkov, L. Venken, S. Dierckx, S. J. Vanderleyden, A. V. Silhanek, C. A. Condat, and V. I. Marconi, "Influence of swimming strategy on microorganism separation by asymmetric obstacles," Phys. Rev. E 87, 052702 (2013).

${ }^{29}$ P. K. Ghosh, P. Hanggi, F. Marchesoni, and F. Nori, "Giant negative mobility of Janus particles in a corrugated channel," Phys. Rev. E 89, 062115 (2014).

${ }^{30} \mathrm{G}$. Volpe, I. Buttinoni, D. Vogt, H.-J. Kümmerer, and C. Bechinger, "Microswimmers in patterned environments," Soft Matter 7, 8810 (2011).

${ }^{31}$ P. E. Kloeden and E. Platen, Numerical Solution of Stochastic Differential Equations (Springer, 1992).

${ }^{32}$ A. Taloni, O. Flomenbom, R. Castaẽda-Priego, and F. Marchesoni, "Single file dynamics in soft materials," Soft Matter 13, 1096 (2017).

${ }^{33}$ Y. Elskens and H. L. Frisch, "Annihilation kinetics in the one-dimensional ideal gas," Phys. Rev. A 31, 3812 (1985).

${ }^{34}$ R. A. Blythe, M. R. Evans, and Y. Kafri, "Stochastic ballistic annihilation and coalescence," Phys. Rev. Lett. 85, 3750 (2000).

${ }^{35}$ S. Chandrasekhar, "Stochastic problems in physics and astronomy," Rev. Mod. Phys. 15, 1 (1943).

${ }^{36}$ G. Miño, T. E. Mallouk, T. Darnige, M. Hoyos, J. Dauchet, J. Dunstan, R. Soto, Y. Wang, A. Rousselet, and E. Clement, "Enhanced diffusion due to active swimmers at a solid surface," Phys. Rev. Lett. 106, 048102 (2011). 